\title{
GLOBALLY ANALYTIC HYPOELLIPTIC VECTOR FIELDS ON COMPACT SURFACES
}

\author{
ADALBERTO P. BERGAMASCO AND SÉRGIO LUÍS ZANI
}

(Communicated by David Tartakoff)

\begin{abstract}
We present a characterization of the global analytic hypoellipticity of a complex, non-singular, real analytic vector field defined on a compact, connected, orientable, two-dimensional, real analytic manifold.

In particular, we show that such vector fields exist only on the torus.
\end{abstract}

\section{INTRODUCTION}

In this work we treat complex, non-singular, real analytic vector fields defined on a compact, connected, orientable, two-dimensional, real analytic manifold $M$. We say that a vector field $L$ is globally analytic hypoelliptic on the manifold $M$ if the conditions $u \in \mathcal{D}^{\prime}(M)$ and $L u \in C^{\omega}(M)$ imply that $u \in C^{\omega}(M)$.

We recall some of the known results. Greenfield in $[\mathrm{Gr}]$ proved among other things that if $L=\alpha \partial_{t}+\beta \partial_{x}$ has constant complex coefficients, then $L$ is globally analytic hypoelliptic on the two-torus if and only if for any $\varepsilon>0$ there exists $C>0$ such that $|\alpha p+\beta q| \geq C \exp (-\varepsilon(|p|+|q|))$, for all $(p, q) \in \mathbb{Z}^{2}$ such that $|p|+|q| \geq C$.

In $[\mathrm{B}]$, the tube case on the two-torus was considered, namely, $L$ is of the form $\partial_{t}+(a(t)+i b(t)) \partial_{x}$ where $a$ and $b$ are real-valued and real analytic functions. It was proved there that $L$ is globally analytic hypoelliptic if and only if the following conditions are satisfied:

(1) $b$ does not change sign;

(2) If $b \equiv 0$, then the real number $a_{0} \doteq(2 \pi)^{-1} \int_{0}^{2 \pi} a(t) d t$ is neither rational nor exponential-Liouville (see Definition 2.1).

The tube case was again taken up in [BZ]; there, the main goal was to give precise results about the location of the singularities of the solutions. For further information on the tube case see [BNZ].

The main goal of the present work is to extend the global analytic hypoellipticity results beyond the tube case and, furthermore, to vector fields defined on manifolds more general than the two-torus.

The smooth case, that is, the global hypoellipticity of smooth vector fields, was completely studied by Hounie in [H2].

Received by the editors January 22, 2007.

2000 Mathematics Subject Classification. Primary 35H10, 58GXX.

Key words and phrases. Complex vector fields, global analytic hypoellipticity, Liouville numbers, sheaf cohomology, Sussmann orbits.

The first author was partially supported by CNPq. Both authors were partially supported by FAPESP. 
Part of our proofs follows the ideas for the smooth case in [H2] which relies heavily on the use of cut-off functions. One of the difficulties of adapting such arguments is that in the analytic case there are no such functions. Instead of that we use the fact that the first cohomology group of the manifold $M$ with coefficients in the sheaf of germs of real analytic functions vanishes, that is, $H^{1}\left(M, C^{\omega}\right)=0$ ([G]). In particular, we will use the following argument: Let $K \subset V \subset M$ where $K$ is compact and $V$ is open. If $u \in \mathcal{D}^{\prime}(V)$ is real analytic in $V \backslash K$ and $w \in C^{\omega}(M \backslash K)$, then $u-w$ is real analytic in $V \backslash K$ and since $H^{1}\left(M, C^{\omega}\right)=0$, we obtain $u_{1} \in C^{\omega}(V)$ and $u_{2} \in C^{\omega}(M \backslash K)$ such that $u_{1}-u_{2}=u-w$ in $V \backslash K$; that is, $u_{2}-w=u_{1}-u$ in $V \backslash K$. This allows us to define the global object $w \in \mathcal{D}^{\prime}(M)$ by

$$
v= \begin{cases}u_{2}-w, & \text { in } M \backslash K, \\ u_{1}-u, & \text { in } V .\end{cases}
$$

Our results make use of the concepts of minimal sets and orbits (in the sense of Sussmann) of the pair of real vector fields $\Re L$ and $\Im L$. We recall their definitions. Let $G$ be the group of diffeomorphisms generated by the one-parameter group whose infinitesimal generators are $\Re L$ and $\Im L$. A set $\Omega \subset M$ is called invariant if $g \Omega \subset \Omega$ for all $g \in G$. A set is said to be minimal if it is closed, invariant, non-empty, and contains no such proper subset. The orbits of $G$ are called orbits of $L$.

In [H1] Hounie proved that $M$ is the only minimal set for a non-singular globally hypoelliptic vector field. The same remains true for non-singular globally analytic hypoelliptic vector fields. Indeed, arguing as in [H1], the only possibilities for a minimal set $\Omega \subset M$ of a non-singular vector field $L$ is either an orbit homeomorphic to $S^{1}$ or $M$ itself. If the former holds, then, since $M$ is orientable, it is possible to define a function $f$ in a tubular neighborhood $V$ of $\Omega$ taking the value 1 on one component of $V \backslash \Omega$ and 0 on the other one. Thus, $L f=0$. We now make use of the cohomology argument with $K=\Omega$, and we obtain a distribution $u \in \mathcal{D}^{\prime}(M) \backslash$ $C^{\omega}(M)$ satisfying $L u \in C^{\omega}(M)$. This shows that if there is an orbit homeomorphic to $S^{1}$, then $L$ cannot be globally analytic hypoelliptic. In particular, if $L$ is a nonsingular globally analytic hypoelliptic vector field, then $M$ is the closure of an orbit of $L$.

We call a non-singular vector field $L$ in $M$ a type I vector field if its real and imaginary parts are linearly dependent everywhere. Otherwise, $L$ will be referred to as a type II vector field.

We deal with each of these two cases separately.

Our results provide a complete answer to the problem under study.

In the type I case we prove that a vector field $L$ is globally analytic hypoelliptic if and only if $L$ can be transformed into a multiple of a constant coefficient vector field on the two-torus satisfying a diophantine condition (see Theorem 2.2). Note that in this case the vector fields are never (locally) analytic hypoelliptic at any point of the manifold.

In the type II case we prove that a vector field $L$ is globally analytic hypoelliptic if and only if the Nirenberg-Treves condition $(\mathcal{P})$ holds and $M$ is the only Sussmann orbit of $L$ (see Theorem 3.1). It turns out that in this case $L$ is globally analytic hypoelliptic if and only if it is (locally) analytic hypoelliptic at every point of the manifold.

It follows from our results that, in the class of compact surfaces, globally analytic hypoelliptic vector fields can exist only on the torus (see Corollary 3.2). 


\section{REAL VECTOR FIELDS}

We begin with a definition.

Definition 2.1. An irrational number $\alpha$ is said to be exponential-Liouville if there exist $\varepsilon>0$ and infinitely many solutions $p / q, p \in \mathbb{Z}, q \in \mathbb{N}$, to the inequality $|\alpha-p / q| \leq \exp (-\varepsilon q)$.

Theorem 2.2. Let $L=X+i Y \neq 0$ be a non-singular real analytic vector field on a compact real analytic surface $M$. Assume that $L$ is type $I$.

Then $L$ is globally analytic hypoelliptic on $M$ if and only if the following conditions hold:

(a) $M$ is real analytically diffeomorphic to $\mathbb{T}^{2}$;

(b) There exist coordinates $x, t$ on $\mathbb{T}^{2}$ such that $L=g(x, t)\left(\partial_{t}+\gamma \partial_{x}\right)$, where $g \neq 0$ everywhere and $\gamma$ is a real number which is neither rational nor exponential-Liouville.

Proof. The sufficiency of (a) and (b) is a direct consequence of the work [Gr].

For the necessity of (a) and (b) we follow the argument in the proof of Theorem $\mathrm{A}$ in [H2]. One of the main difficulties of doing this is that we are not allowed to use cut-off functions in order to construct singular solutions. As a replacement we use the fact that $H^{1}\left(M, C^{\omega}\right)=0$.

Another aspect in the proof of Theorem A in [H2] is the fact that in several instances smooth objects are constructed while for our proof we need the real analytic analogues. The latter are obtained by means of a theorem of Grauert and Remmert, as stated in $[\mathrm{H}]$, which yields the approximation of smooth objects such as maps, embeddings and diffeomorphisms by real analytic ones.

We will repeat part of the arguments in [H2].

We begin by using a real analytic approximation of a smooth partition of unity together with a real analytic version of arguments in [H1] and by using the assumption that $L$ is globally analytic hypoelliptic. We obtain a real non-singular vector field, $Z$, and functions $\alpha$ and $\beta$ of class $C^{\omega}$ such that $X=\alpha Z, Y=\beta Z$, $L=(\alpha+i \beta) Z$, with $\alpha+i \beta \neq 0$ everywhere in $M$. In particular, $M$ is homeomorphic to the torus $\mathbb{T}^{2}$; in this proof we consider $\mathbb{T}^{2} \doteq \mathbb{R}^{2} / \mathbb{Z}^{2}$.

Arguing as in [H2] we find a real analytic coordinate $t=t(p)$, and a mod 1 continuous function $\theta=\theta(p)$, such that $p \rightarrow(\theta(p), t(p))$ is a homeomorphism of $M$ onto the torus $\mathbb{T}^{2}$ and there exists an irrational number $\gamma$ such that $\theta(p)-\gamma t(p)$ is constant on each integral curve of $Z$.

In our case we must show that $\theta$ is real analytic and $\gamma$ is not an exponentialLiouville number.

Let $\Sigma=\{p ; \theta(p)=0\}$.

As in [H2] the coordinate $\theta$ gives rise to a distribution $\phi \in \mathcal{D}^{\prime}\left(\mathbb{T}^{2}\right)$ with the following properties:

- $\phi \in C^{0}\left(\mathbb{T}^{2} \backslash \Sigma\right)$;

- $\phi$ has jump equal to 1 at $\theta(p)=0$;

- $L \phi=\delta_{\Sigma}+\{L(\phi)\}$,

where $\delta_{\Sigma}$ is a measure concentrated at $\Sigma$.

Let $\Sigma \subset V \subset \mathbb{T}^{2}$ be a tubular neighborhood of $\Sigma$.

Let $u \in \mathcal{D}^{\prime}(V)$ be such that $u=0$ on one side of $\Sigma$ and $u=1$ on the other side. Clearly, $u$ is real analytic in $V \backslash \Sigma$. 
At this point [H2] uses a cut-off function in order to produce a singular solution. We must proceed in a different way. Since $H^{1}\left(\mathbb{T}^{2}, C^{\omega}\right)=0$, we get $u_{1} \in C^{\omega}(V)$ and $u_{2} \in C^{\omega}\left(\mathbb{T}^{2} \backslash \Sigma\right)$ such that $u_{1}-u_{2}=u$ in $V \backslash \Sigma$, that is, $u_{2}=u_{1}-u$ in $V \backslash \Sigma$.

We define $v \in \mathcal{D}^{\prime}\left(\mathbb{T}^{2}\right)$ by means of

$$
v=\left\{\begin{array}{l}
u_{2}, \quad \text { in } \mathbb{T}^{2} \backslash \Sigma, \\
u_{1}-u, \quad \text { in } V .
\end{array}\right.
$$

Note that $v$ satisfies the following properties:

- $v \in C^{\omega}\left(\mathbb{T}^{2} \backslash \Sigma\right)$,

- $v$ has a jump equal to 1 at $\theta(p)=0$,

- $L v=\delta_{\Sigma}+\{L(v)\}$.

We now resume the reasoning in [H2]. Since $L$ is globally analytic hypoelliptic and $L(\phi-v) \in C^{\omega}\left(\mathbb{T}^{2}\right)$, one has $\phi-v \in C^{\omega}\left(\mathbb{T}^{2}\right)$, which implies that $\phi$, and hence, $\theta$ is real analytic in $\mathbb{T}^{2} \backslash \Sigma$. By using another cycle $\Sigma^{\prime}$, we see that $\theta \in C^{\omega}\left(\mathbb{T}^{2}\right)$.

Thus, $L$ is equivalent to a multiple of $\partial_{t}+\gamma \partial_{\theta}$. It follows from $[\mathrm{Gr}]$ that the irrational number $\gamma$ is not exponential-Liouville. The proof is complete.

\section{NON-REAL VECTOR FIELDS}

We now consider vector fields of type II; the version concerning global hypoellipticity is also due to Hounie in [H2].

Recall the Nirenberg-Treves condition $(\mathcal{P})$. Let $P(x, D)$ be a differential operator defined on a manifold $M$, and let $p(x, \xi)$ denote the principal symbol of $P(x, D)$. Then $P(x, D)$ is said to satisfy condition $(\mathcal{P})$ on $M$ if there is no complex-valued, smooth function $q(x, \xi)$ on $T^{*} M \backslash 0$ such that $\Im(q p)$ takes both positive and negative values on a bicharacteristic of $\Re(q p)$ where $q \neq 0$. Recall that a bicharacteristic of $\Re(q p)$ is an integral curve of the Hamilton field of $\Re(q p)$ over which $\Re(q p)$ vanishes.

If $L$ is a complex vector field on $M$, then $L$ satisfies condition $(\mathcal{P})$ if and only if the following two properties are verified (see [H2] and [T]):

(i) The orbits of $L$ in $M$ have dimension at most two;

(ii) The orbits of $L$ of dimension two are orientable and $\operatorname{Re} L \wedge \operatorname{Im} L$ does not change sign on each such orbit.

If $L$ is a non-singular vector field on a two-dimensional, orientable, manifold $M$ and if $M$ is the only orbit, then $L$ satisfies $(\mathcal{P})$ if and only if $\operatorname{Re} L \wedge \operatorname{Im} L$ does not change sign on $M$.

Theorem 3.1. Let $L=X+i Y \neq 0$ be a non-singular real analytic vector field on a compact, orientable, real analytic surface $M$. Assume that $L$ is of type II. Then $L$ is globally analytic hypoelliptic on $M$ if and only if the following properties are verified:

(a) The Nirenberg-Treves condition $(\mathcal{P})$ holds in $M$;

(b) $L$ has precisely one orbit, namely, $M$.

Furthermore, if $M$ carries a non-singular globally analytic hypoelliptic vector field of type II, then $M$ is real analytically diffeomorphic to the torus $\mathbb{T}^{2}$.

Proof. Necessity of (b). For a non-singular vector field $L$ a minimal set is either homeomorphic to $\mathbb{T}^{1}$ or equal to all of $M$ (see Theorem 1 in [H2]). Recall from the Introduction that for a globally analytic hypoelliptic vector field there can be no minimal set homeomorphic to $\mathbb{T}^{1}$. 
Take $p$ such that $X_{p}$ and $Y_{p}$ are linearly independent. Then the orbit $\gamma(p)$ containing $p$ is a two-dimensional orbit, hence it is a non-empty open set. Since its closure, $\overline{\gamma(p)}$, is invariant and $M$ is minimal, we have $\overline{\gamma(p)}=M$. Now, if we had $\overline{\gamma(p)} \backslash \gamma(p) \neq \varnothing$, then the boundary of $\gamma(p)$, which is invariant, would contain a minimal set different from $M$. Thus, $\gamma(p)=\overline{\gamma(p)}=M$; in other words, $M$ is the only orbit.

Necessity of (a), that is, necessity of condition $(\mathcal{P})$. We are going to use the fact that $M$ is the only orbit, as we have just proved. We proceed by contradiction. Assume that condition $(\mathcal{P})$ does not hold. Then there are $q \neq 0$ and an arc of bicharacteristic of $\Re(q L)$ along which $\Im(q L)$ changes sign. Since our vector field is real analytic, there is a point $\left(A, \xi_{0}\right)$ on the bicharacteristic so that the change of sign occurs precisely at such a point. We are now in a position to use Proposition 2.6 in $[\mathrm{CH}]$ to obtain an open neighborhood, $U$, of $A$ and a function $u \in C^{\infty}(U)$ satisfying $L u=0$, with $\Re u$ having a strict minimum at a point $p \in U$. Inspection of the proof shows that, in fact, $u \in C^{\omega}(U)$. In more detail, the proof uses the preparation theorem, the Cauchy-Kowalevski Theorem, Taylor series and changes of variables arising from analytic ODEs, all of which yield analytic objects. Now one uses a branch of the square root to manufacture a local singular solution, that is, $v \in \mathcal{D}^{\prime}(U)$ such that $\operatorname{sing} \operatorname{supp}_{\mathrm{A}}(v)=\{p\}$ and $L v \in C^{\omega}(U)$. Finally, the cohomological argument shows that there is a global singular solution, that is, $v \in \mathcal{D}^{\prime}\left(\mathbb{T}^{2}\right)$ such that $\operatorname{sing} \operatorname{supp}_{\mathrm{A}}(v)=\{p\}$ and $L v \in C^{\omega}\left(\mathbb{T}^{2}\right)$. This contradicts the assumption that $L$ is globally analytic hypoelliptic.

Sufficiency of (a) and (b). Let $u \in \mathcal{D}^{\prime}(M)$ be such that $L u \in C^{\omega}(M)$. Let $p \in M$. We will show that $u$ is real analytic in some neighborhood of $p$.

By working in a local chart centered at $p$, we may assume that our vector field is defined in a neighborhood of the origin in $\mathbb{R}^{2}$. Thus, we may proceed as in [BT1] and choose a small rectangle, $\mathcal{R}=J \times I$, centered at the origin where a first integral of the form $Z(x, t)=x+i \varphi(x, t)$, with $\varphi$ real-valued and $\varphi(x, 0) \equiv 0$, is defined. Locally, $L$ is a non-vanishing multiple of

$$
L_{o}=Z_{x} \partial_{t}-Z_{t} \partial_{x}=\left(1+i \varphi_{x}\right) \partial_{t}-i \varphi_{t} \partial_{x}
$$

and $\operatorname{Re} L \wedge \operatorname{Im} L$ is a non-vanishing multiple of

$$
\operatorname{Re} L_{o} \wedge \operatorname{Im} L_{o}=-\varphi_{t} \partial_{t} \wedge \partial_{x} .
$$

As we mentioned before, our hypotheses (a) and (b) imply that $\operatorname{Re} L \wedge \operatorname{Im} L$ does not change sign on $M$, hence, $\varphi_{t}$ does not change sign on $\mathcal{R}$, say, $\varphi_{t} \geq 0$.

We cannot have $\varphi(0, \cdot) \equiv 0$ in $(-r, r) \subset I, r>0$. Indeed, if this were not the case then, by analyticity, the origin would belong to a one-dimensional orbit, violating (b). Hence, $t=0$ is a zero of odd order of $\varphi(0, \cdot)$. Therefore, condition (c) of Theorem 2.6 of [BT1] does not hold and, consequently, the non-validity of condition (a) of the same theorem implies that $u$ is real analytic near the origin.

Finally, assume that on the surface $M$ there is a non-singular, non-real vector field $L=X+i Y$ such that $M$ is the only orbit of $L$ and $X \wedge Y$ does not change sign over $M$. Then, an argument in pages 361-362 in [H2], building up on the real vector fields $X$ and $Y$, yields a real, non-singular vector field on $M$; this shows that the Euler characteristic of $M$ is zero and $M$ has to be homeomorphic (and hence real analytically diffeomorphic) to the two-torus. The proof is complete.

As a consequence of Theorems 2.2 and 3.1 we have 
Corollary 3.2. If there exists a non-singular, globally analytic hypoelliptic vector field on a compact surface $M$, then $M$ is (real analytically diffeomorphic to) the two-torus.

\section{ACKNOWLEDGEMENTS}

This paper is in part motivated by another work which we had started jointly with Jean-Marie Trépreau to whom we are grateful for his generosity.

\section{REFERENCES}

[BT1] Baouendi, M. S. and Trèves, F., A local constancy principle for the solutions of certain overdetermined systems of first-order linear partial differential equations, Adv. in Math. Suppl. Stud., 7a, Academic Press, New York-London, (1981), 245-262. MR634241 (84f:35105)

[B] Bergamasco, A. P., Remarks about global analytic hypoellipticity, Trans. Amer. Math. Soc. 351 (1999), no. 10, 4113-4126. MR1603878 (99m:35032)

[BNZ] Bergamasco, A., Nunes, W. and Zani, S., Global properties of a class of overdetermined systems, J. Funct. Anal. 200 (2003), no. 1, 31-64. MR1974087 (2004c:35295)

[BZ] Bergamasco, A. and Zani, S., Prescribing analytic singularities for solutions of a class of vector fields on the torus, Trans. Amer. Math. Soc. 357 (2005), no. 10, 4159-4174. MR2159704 (2006c:35002)

[CH] Cordaro, P. and Hounie, J., On local solvability of underdetermined systems of vector fields, Amer. J. Math. 112 (1990), 243-270. MR1047299 (91j:58150)

[G] Grauert, H., On Levi's problem and the imbedding of real-analytic manifolds, Ann. of Math. (2) 68 (1958), 460-472. MR0098847 (20:5299)

[Gr] Greenfield, S. J., Hypoelliptic vector fields and continued fractions, Proc. Amer. Math. Soc. 31 (1972), 115-118. MR0301459 (46:617)

[H] Hirsch, M., Differential topology, Graduate Texts in Mathematics, No. 33, Springer-Verlag, New York, 1976. MR0448362 (56:6669)

[H1] Hounie, J., Minimal sets of families of vector fields on compact surfaces, J. Differential Geom. 16 (1981), no. 4, 739-744. MR661663 (83j:58075)

[H2] Hounie, J., Globally hypoelliptic vector fields on compact surfaces, Comm. Partial Differential Equations 7 (1982), no. 4, 343-370. MR652813 (83k:35025)

[S] Sussmann, H., Orbits of families of vector fields and integrability of distributions. Trans. Amer. Math. Soc. 180 (1973), 171-188. MR0321133 (47:9666)

[T] Treves, F., Hypo-analytic Structures, Princeton University Press, Princeton, NJ, 1992. MR1200459 (94e:35014)

Departamento de Matemática, Instituto de Ciências Matemáticas e de ComputaÇão - USP, Caixa Postal 668, SÃo Carlos, SP, 13560-970, Brasil

E-mail address: apbergam@icmc.usp.br

Departamento de Matemática, Instituto de Ciências Matemáticas e de ComputaÇão - USP, Caixa Postal 668, São Carlos, SP, 13560-970, Brasil

E-mail address: szani@icmc.usp.br 